\title{
En revenant des Assises des États Généraux de la Recherche de Grenoble
}

\section{Hervé Chneiweiss}

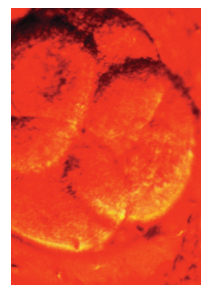

Grenoble sous la pluie en cette veille de Toussaint 2004, un peu plus de 300 jours depuis le début du mouvement SLR (Sauvons la Recherche) et une fabuleuse aventure humaine jalonnée de plusieurs succès non négligeables: le rétablissement des postes 2004 pour les jeunes dans les organismes et les universités, un arrêt de l'hémorragie budgétaire, et ces États Généraux. Quelques chiffres pour donner le vertige: 1500 rédacteurs, 34 contributions principales au débat issues des Comités locaux (CLOEG), 1 million de visites en 3 mois sur le site des États Généraux, 225000 signatures citoyennes à la lettre SLR en plus des 75000 scientifiques, 57000 téléchargements du texte final [1]. Mais, à Grenoble, aucun raton laveur, tout au plus la crème du monde de l'administration de la recherche (organismes, universités, grandes écoles, grands patrons...) et les principaux responsables de partis politiques: François Hollande (PS), François Bayrou (UDF), Marie-Georges Buffet (PC), Marie-Christine Blandin (Verts), Roger-Gérard Schwartzenberg (PRG), tandis que Nicolas Sarkozy (UMP) s'excusait à la dernière minute. Une présence qui illustrait à merveille la proposition faite par le Comité ERMES de l'Inserm en mars 2004: «Parce que toute activité de recherche est exploration de l'inconnu et remise en question des certitudes, le soutien qu'une société décide d'accorder à la recherche traduit la manière dont cette société vit sa relation à l'avenir. [...] L'engagement pour la recherche, au même titre que l'engagement pour l'enseignement, la solidarité sociale, les soins et la lutte contre les inégalités représente, avant tout, un choix de société - un engagement sur des valeurs » $[2,3]$. Comme un écho à ce texte, le résumé du rapport final commence par ces mots: «Les propositions des États Généraux s'adressent à l'ensemble des citoyens. Elles ont pour dessein de recomposer les rapports des acteurs de la recherche publique avec la société, dans laquelle ils remplissent cinq missions: l'élaboration, la diffusion des connaissances, la formation à et par la recherche, la valorisation des résultats de la recherche et l'expertise. Au cœur de ces activités, la connaissance scientifique possède le caractère particulier d'un bien public dont l'État est le principal promoteur et dont il est le garant $\gg$.

Plus d'un millier de personnes ont débattu ensuite pendant deux jours. Nous renvoyons au texte intégral [1] dont il serait illusoire de chercher à faire ici un résumé. Nous en rappellerons donc simplement les lignes de force. La première est l'affirmation que l'élaboration des connaissances scientifiques est la première mission de la recherche scientifique, que la connaissance scientifique doit être reconnue comme un bien public mondial, également accessible aux pays du Sud. Cela implique en corollaire une forte implication du financement public de cette recherche, assortie d'une évaluation rigoureuse.

La seconde ligne de force est la déclinaison des moyens nouveaux nécessaires à la refondation de la recherche en France: (1) des moyens financiers, représentés par une augmentation régulière d'un milliard d'euros chaque année, au moins pour les 5 ans à venir; (2) la programmation pluriannuelle du budget et de l'emploi, associée à une augmentation du nombre d'emplois publics, et la résorption de la précarité sont des priorités; (3) de nouveaux outils de conseil et de gouvernance avec le Haut Conseil de la Science placé auprès de l'autorité politique et le Comité d'Évaluation des Opérateurs de Recherche (CEOR) pour un audit régulier de la politique scientifique des opérateurs de recherche.

Un troisième axe de réformes s'adresse à la structure même des opérateurs de recherche et appelle à une profonde réorganisation de l'Université, des grandes écoles et des 
organismes, pour placer une recherche bien évaluée et bien coordonnée entre les différents acteurs, au cœur de leurs activités. Pour les unités de recherche, une simplification des tâches administratives et des modalités de financements, une pluri-annualité des budgets, un niveau de financement de base de ces budgets à hauteur de $70 \%$ de leurs besoins de fonctionnement, sont parmi les propositions les plus urgentes à mettre en œuvre. L'évaluation rigoureuse des projets et de l'activité en étant le corollaire. Inutile de parler de recherche sans évoquer le sort des chercheurs. Précarité et « travail au noir » caractérisent aujourd'hui une partie importante de l'entrée dans la recherche. Ce n'est plus acceptable et un cadre est maintenant proposé, pour la thèse et la période immédiatement après. Cela implique ici encore une programmation pluri-annuelle de l'emploi pour donner une visibilité à la carrière scientifique, aujourd'hui en danger comme en témoigne la dramatique diminution des inscriptions en troisième cycle scientifique. La question du corps unique, enseignants-chercheurs, a été tranchée dans le sens de la diversité et des passerelles, mais la réforme universitaire devra permettre de libérer un plus grand temps pour la recherche, surtout pour les plus jeunes.

Enfin, comment parler aujourd'hui d'une politique nationale de la recherche sans l'intégrer à sa dimension naturellement européenne? Outre l'objectif communautaire des $3 \%$ de PIB investis en R\&D, la création du Conseil européen de la recherche (ERC) dont la mission sera de financer des projets de recherche fondamentale sur la base de leur qualité scientifique et de leur contribution, par des partenariats, à la construction européenne, devra être assortie d'une modification des règles de gestion des programmes européens afin de leur assurer souplesse et réactivité.

Est-ce fini? Non, surtout pas. Grenoble constitue la fin du début. II faut maintenant mettre en œuvre et cette réforme demandera bien plus qu'une Loi d'Orientation et de Programmation de la Recherche, par exemple une profonde réforme des universités et du système des grandes écoles. La rédaction des décrets sera d'une extrême importance également. Et puis, il nous reste à travailler de nombreux points encore trop généraux, comme les modalités de l'évaluation, ou la gouvernance des organismes de recherche et le rôle de leurs conseils scientifiques. Bref, de grandes perspectives pour une recherche française encore à sauver!

Back from the States General

of Research conference in Grenoble

\section{RÉFÉRENCES}

1. Le rapport complet est disponible sur le site du CIP (http://cip-etatsgeneraux.apinc.org).

2. Le texte complet du comité ERMES est disponible sur le site de l'Inserm (www.inserm.fr).

3. Debuire B, Hirsch $\varepsilon$ (sous la direction de). La Recherche peut-elle se passer d'éthique? Paris: Espace Éthique-AP-HPTIRÉS À PART Vuibert, 2004. 